\title{
Infecciones asociadas a sistemas de derivación de líquido cefalorraquídeo en pediatría: análisis epidemiológico y de factores de riesgo de mortalidad
}

\author{
Cerebrospinal fluid shunt-associated infections in pediatrics: \\ Analysis of the epidemiology and mortality risk factors
}

\author{
Dra. Soledad González ${ }^{a}$ Lic. Mirta Carbonaro ${ }^{a}$, Dra. Ana G. Fedullo ${ }^{a}$, \\ Dra. María I. Sormani ${ }^{a}$, Bioq. María del C. Ceinos ${ }^{b}$, Dr. Roberto González ${ }^{c}$ y \\ Dra. María T. Rosanova ${ }^{a}$
}

\section{RESUMEN}

Introducción. La infección es de las complicaciones más frecuentes de los sistemas de derivación ventricular de líquido cefalorraquídeo. El objetivo fue describir las características clínicas, microbiológicas y evolutivas de niños con infección asociada a sistemas de derivación ventricular de líquido cefalorraquídeo y analizar los factores de riesgo, relacionados con la mortalidad.

Población y métodos. Estudio descriptivo, retrospectivo, llevado a cabo en el Hospital "Prof. Dr. Juan P. Garrahan" de la Ciudad de Buenos Aires. Se evaluaron todos los pacientes internados desde el 1/1/2012 y el 31/12/2015 compatibles con ventriculitis y cultivo de líquido cefalorraquídeo positivo.

Resultados. Se incluyeron 49 pacientes con 57 infecciones. La mediana de edad fue de 62 meses (rango intercuartílico: 19-114). Predominó el sexo masculino: $34(70 \%)$. El tumor del sistema nervioso central fue la enfermedad de base más frecuente: $20(40 \%)$. Se aisló estafilococo coagulasa negativo en 26 (46\%), Staphylococcus aureus en $13(23 \%)$, bacilos Gramnegativos en $11(19 \%)$ y otros en $7(12 \%)$. En $55(97 \%)$ de las infecciones, se realizó tratamiento quirúrgico con retiro del sistema de derivación ventricular más antibioticoterapia. La mortalidad fue del 9\%. Los únicos factores asociados a la mortalidad estadísticamente significativos fueron hemocultivos positivos $(\mathrm{p}=0,04)$, fiebre al ingreso $(\mathrm{p}=0,04)$ y shock séptico $(\mathrm{p}=0,0006)$. Conclusiones. El estafilococo coagulasa negativo fue el germen más frecuente. El retiro de la válvula, junto con la antibioticoterapia, fue el tratamiento más utilizado. La presencia de fiebre al ingreso, hemocultivos positivos y shock séptico fueron predictores de mortalidad.

Palabras clave: ventriculitis cerebral, mortalidad, niño, derivaciones del líquido cefalorraquídeo.

http: / / dx.doi.org/10.5546/ aap.2018.198 Texto completo en inglés: http:/ / dx.doi.org/10.5546/ aap.2018.eng.198
Cómo citar: González S, Carbonaro M, Fedullo AG, et al. Infecciones asociadas a sistemas de derivación de líquido cefalorraquídeo en pediatría: análisis epidemiológico y de factores de riesgo de mortalidad. Arch Argent Pediatr 2018;116(3):198-203.

\section{INTRODUCCIÓN}

Los sistemas de derivación ventricular de líquido cefalorraquídeo (SDV-LCR) son frecuentemente utilizados en neurocirugía pediátrica para el tratamiento de la hidrocefalia de diversas etiologías, entre las que se incluyen alteraciones anatómicas (mielomeningocele, ArnoldChiari), prematurez con hemorragia intraventricular, infecciones del sistema nervioso central (SNC), tumores o un trauma craneoencefálico grave. ${ }^{1}$

La infección es una de las principales causas de morbimortalidad ${ }^{2}$ en niños que son sometidos a procedimientos de colocación de SDV-LCR.

La incidencia reportada es variable: desde $0,3 \%$ a $26 \% .^{3}$ Esta complicación se asocia con el requerimiento de múltiples procedimientos quirúrgicos, la prolongación de los tiempos de internación y el aumento de los costos hospitalarios. Existen cuatro mecanismos propuestos que favorecerían la infección: el primero y más frecuente es la colonización del sistema durante el acto quirúrgico; el segundo mecanismo es la infección retrógrada desde el extremo distal, por ejemplo, en una perforación 
intestinal; el tercer mecanismo es a través de la piel, luego de la punción del reservorio o por una lesión de la piel que recubre el catéter; y el cuarto mecanismo es hematógeno, en general, en pacientes con derivaciones ventrículo-atriales que padecen bacteriemias. ${ }^{4}$

Los factores asociados a mayor riesgo de infecciones de los SDV-LCR incluyen prematurez, menor edad, infección previa, etiología de la hidrocefalia (meningitis purulenta, mielomeningocele y hemorragias), menor experiencia del cirujano, la duración del procedimiento quirúrgico, inadecuada preparación de la piel y las revisiones del sistema frecuentes, ${ }^{4}$ entre otras.

Los microorganismos causantes de las infecciones asociadas a los SDV-LCR son, en general, poco virulentos, producen escasa reacción inflamatoria y se adhieren a la superficie del dispositivo y forman biofilms, lo cual dificulta el diagnóstico y el tratamiento. Los síntomas son frecuentemente inespecíficos; en la mayoría de los casos, solo están presentes los síntomas atribuibles a la hipertensión endocraneana generada por la disfunción valvular (cefalea, náuseas, vómitos y alteración del estado mental). ${ }^{5}$

La mortalidad reportada es variable, en general, baja, aunque existen publicaciones recientes con una mortalidad mayor del $15 \% .2,6$ Hay pocos estudios en pediatría que evalúen los factores asociados a la mortalidad en este tipo de infecciones.

\section{OBJETIVO}

Describir las características epidemiológicas, clínicas, microbiológicas y evolutivas de niños con infección asociada a los SDV-LCR y analizar los factores de riesgo relacionados con la mortalidad.

\section{MATERIALES Y MÉTODOS}

En el Hospital de Pediatría "Prof. Dr. Juan P. Garrahan" de la Ciudad de Buenos Aires, en el período comprendido entre el 1/1/2012 y el $31 / 12 / 2015$, se realizó este estudio retrospectivo, descriptivo y observacional.

El presente estudio se encuentra aprobado por el Comité de Ética del Hospital "Prof. Dr. Juan P. Garrahan" de la Ciudad de Buenos Aires.

Criterios de inclusión: niños de 1 mes a 18 años con clínica compatible con ventriculitis y cultivo de LCR positivo. Se consideró clínica compatible con ventriculitis cefalea, vómitos, alteración del estado mental, irritabilidad, fiebre sin otra causa, eritema en el trayecto de una válvula y signos peritoneales sin otra causa en pacientes con derivaciones ventrículoperitoneales.

Recolección de datos: en el contexto de la vigilancia epidemiológica llevada a cabo por un grupo de enfermeras especializadas en el control de infecciones, se registran todos los pacientes que se someten a procedimientos neuroquirúrgicos y se revisan anualmente las tasas de infecciones posquirúrgicas con el objetivo de mejorar la calidad de atención. A partir de dicha base de datos, se identificaron aquellos pacientes que presentaron una infección asociada a SDV-LCR. Posteriormente, se obtuvieron, mediante la revisión de las historias clínicas, datos demográficos, enfermedad de base, etiología de la hidrocefalia, edad del primer SDV-LCR, fecha de colocación y características del shunt actual, características clínicas y microbiológicas del LCR y los hemocultivos, evolución clínica y tratamiento médico y quirúrgico.

Las muestras de LCR se procesaron según el manual de procedimientos del Laboratorio de Microbiología Clínica y se cumplió con las normas (ISO 15189:2007) y las recomendaciones internacionales. ${ }^{7}$ Se realizó previamente un examen microscópico directo (tinción de Gram) y luego un cultivo, con la centrifugación previa. Los medios utilizados para el cultivo fueron sólidos (agar sangre, agar chocolate). Las marcas comerciales empleadas para la preparación de los medios de cultivo fueron Columbia Blood Agar Base Sangre de OXOID para las placas de agar sangre y chocolate $\mathrm{y}$, para el medio líquido (caldo infusión cerebro corazón BHI), Biokar (Francia). Se incubaron los medios sólidos durante $48 \mathrm{~h}$ a temperatura y atmósfera adecuadas, y los medios líquidos, durante 7 días a $35-37^{\circ} \mathrm{C}$. Los cultivos positivos se procesaron mediante el uso del sistema automatizado VITEK ${ }^{\circledR} 2 \mathrm{C}$ (bioMérieux, Francia), y se obtuvieron el género y la especie de los microorganismos aislados, como así también la sensibilidad a los antibióticos por concentración inhibitoria mínima (CIM). ${ }^{8}$

Definiciones: Infección asociada a SDV-LCR fue definida como la identificación de un patógeno bacteriano o fúngico aislado en el LCR obtenido por punción del reservorio de los SDV-LCR o intraquirúrgica, más clínica compatible con ventriculitis.

Mortalidad: se evaluó la ocurrida dentro de los 30 días del diagnóstico de la infección.

Análisis estadístico: Los datos fueron procesados en la base Epi Info versión 6.0. 
Las variables continuas se informaron como mediana y rango intercuartílico (RIC). Las variables categóricas, como número y porcentaje. Se tomó como valor de corte para diferencias estadísticamente significativas un valor de $\mathrm{p} \leq$ de 0,05 .

Para evaluar los factores de riesgo de mortalidad, se realizó el análisis univariado de las variables estudiadas.

\section{RESULTADOS}

Durante el período estudiado, se colocaron un promedio de 261 SDV-LCR por año (rango: 252278). Cumplieron con los criterios de inclusión 49 pacientes, y 8 presentaron 2 infecciones en el período estudiado, por lo cual se registraron 57 infecciones. La mediana de edad fue de 62 meses (RIC: 19-114). Predominó el sexo masculino: 34 pacientes (70\%). Las causas de la hidrocefalia se describen en la Tabla 1. La mediana de días de colocación del sistema hasta la infección fue de 25 días (RIC: 7-37); se subdividieron en 4 períodos: 0-30 días, 33 pacientes (57\%); 31-60 días, 13 pacientes (22\%); 61-90 días, 2 pacientes $(4 \%)$ y $>91$ días, 6 pacientes $(10 \%)$.

La mediana de edad de colocación del primer SDV-LCR fue de 12 meses (RIC: 1-96); requirieron recambio valvular previo 32 pacientes $(56 \%)$ y tenían antecedente de pioventriculitis 17 pacientes $(30 \%)$.

Respecto de las infecciones, en 52 (91\%), el SDV correspondía a derivaciones ventrículoperitoneales; $2(4 \%)$, a derivación ventrículoatrial; y $3(4 \%)$, a otros. La disfunción valvular en $44(77 \%)$, la fiebre en $27(47 \%)$ y la celulitis del trayecto en $6(11 \%)$ fueron las formas de presentación más frecuentes. La media de duración de la fiebre fue 2 días (rango: 1-14). El LCR al ingreso fue patológico en $42(78 \%)$, con una mediana de glucosa,

TABLA 1. Causa de la hidrocefalia ( $n=49$ pacientes)

\begin{tabular}{ll}
\hline & $\mathbf{N}(\%)$ \\
\hline - Tumor del SNC & $20(40)$ \\
- Hidrocefalia congénita & $10(20)$ \\
- Malformaciones anatómicas & $6(12)$ \\
- Meningitis & $5(10)$ \\
- Hemorragia intraventricular & $4(8)$ \\
- Trauma craneoencefálico grave & $2(4)$ \\
- Accidente cerebrovascular & $1(2)$ \\
\hline
\end{tabular}

SNC: sistema nervioso central. celularidad y proteínas de $48 \mathrm{mg} / \mathrm{ml}$ (RIC: 31 62), 28,5 células $/ \mathrm{mm}^{3}$ (RIC: $4-180$ ) y $74 \mathrm{mg} /$ dl (RIC: 21-179), respectivamente. La tinción de Gram fue positiva en 6 pacientes (10\%). Los aislamientos microbiológicos en el LCR fueron estafilococo coagulasa negativo en $26(46 \%)$, Staphylococcus aureus meticilino resistente en 8 (14\%), Staphylococcus aureus meticilino sensible en $5(9 \%)$, Pseudomonas aeruginosa en $3(5 \%)$, Enterobacter spp. en $2(3 \%)$, Pseudomonas stutzeri en $2(3 \%)$, Acinetobacter baumannii en $1(2 \%)$, Stenotrophomonas maltophilia en $1(2 \%)$, Klebsiella pneumoniae en $1(2 \%)$, Achromobacter xylosoxidans en $1(2 \%)$, Candida albicans en $1(2 \%)$ y otros en $6(10 \%)$. En una infección $(2 \%)$, se constató cultivo polimicrobiano. Se halló bacteriemia concomitante con el mismo microorganismo aislado en el LCR en seis (10\%) de los episodios. Los aislamientos en hemocultivos fueron estafilococo coagulasa negativo en $2(3 \%)$, Staphylococcus aureus meticilino resistente en $2(3 \%)$, Staphylococcus aureus meticilino sensible en $1(2 \%)$ y Achromobacter xylosoxidans en $1(2 \%)$.

La mediana de días de internación fue de 35 días (RIC: 23-61). En 55 (97\%) de las infecciones, se realizó el tratamiento quirúrgico con retiro del SDV más antibioticoterapia. Requirieron SDV externa $54(94 \%)$ con mediana de 1 recambio (rango: 1-3) (Tabla 2). Cabe aclarar que, en nuestro Hospital, como parte del protocolo de manejo de los drenajes ventriculares externos (DVE), se realiza el recambio del sistema cada 7 días, aunque algunos pacientes pueden requerir mayor número de recambios por complicaciones mecánicas o infecciosas.

El antibiótico más utilizado fue la vancomicina en $28(49 \%)$. La media de tratamiento fue de 21 días (rango: 14-26). Dos (3\%) de los pacientes

TABLA 2. Evolución y tratamiento

\begin{tabular}{lc}
\hline & N $(\%)$ \\
\hline Días de internación & 35 (RIC: $23-61)$ \\
Retiro del SDV más antibioticoterapia & $55(97)$ \\
SDV externa & $54(94)$ \\
Recolocación del SDV & $46(80)$ \\
Mediana de recambios del & 1 (RIC: $1-3)$ \\
$\begin{array}{l}\text { SDV externa } \\
\text { Mediana de días de }\end{array}$ & 21 (RIC: $14-26)$ \\
tratamiento antibiótico & \\
$\begin{array}{l}\text { Mediana de días hasta la } \\
\text { recolocación del SDV }\end{array}$ & 23 (RIC: $18-30)$ \\
Mortalidad & $5(9)$ \\
\hline
\end{tabular}

RIC: rango intercuartílico;

SDV: sistema de derivación ventricular. 
recibieron tratamiento médico únicamente, ambos presentaban diagnóstico de progresión tumoral y se encontraban en cuidados paliativos.

Luego del tratamiento, a 46 pacientes $(80 \%)$ se les colocó un SDV. La mediana de días hasta la recolocación del SDV fue de 23 días (RIC: 18-30). Respecto a las complicaciones, 3 pacientes $(5 \%)$ presentaron sobreinfección por bacilos Gramnegativos y 5 pacientes $(9 \%)$ fallecieron a causa de la infección. Dos pacientes fallecieron dentro de las 48 horas del ingreso por shock séptico; ambos presentaron hemocultivos positivos con aislamiento de Staphylococcus aureus. Los tres pacientes restantes fallecieron dentro del mes del diagnóstico de la ventriculitis, sin control de la infección al momento del deceso. Presentaban como enfermedad de base un tumor del SNC con diagnóstico de recaída concomitante.

En el análisis univariado, se encontraron como únicos factores asociados a la mortalidad estadísticamente significativos la presencia de hemocultivos positivos $(p=0,04)$, fiebre al ingreso $(\mathrm{p}=0,04)$ y shock séptico $(\mathrm{p}=0,0006)$.

\section{DISCUSIÓN}

La infección es una de las complicaciones más frecuentes de las derivaciones ventriculares del LCR. ${ }^{1}$ La incidencia reportada de las ventriculitis asociadas al acto quirúrgico varía desde el $1 \%$ al $23 \%{ }^{3}$ con una disminución en los últimos años luego de la introducción de diversas estrategias para la prevención de las infecciones de sitio quirúrgico (ISQ). En Estados unidos y Canadá, reportan tasas menores del 5,7\%. ${ }^{9}$ En cambio, en otros países, como Turquía, reportan tasas del $15,8 \%{ }^{10}$ y Korea, del 10,5\%. ${ }^{11}$ En nuestro Hospital, las tasas de infección registradas fueron $4,37 \%, 5,16 \%, 3,41 \%$ y $6,5 \%$ para $l o s$ años 2012, 2013, 2014 y 2015, respectivamente. La amplia variabilidad de tasas reportadas en la literatura se ve influenciada por los distintos criterios diagnósticos utilizados en cada estudio. En algunos trabajos, incluido el nuestro, ${ }^{11-13}$ se considera únicamente la positividad del LCR, mientras que otros incluyen cultivos y/o alteraciones del citoquímico. ${ }^{5,9,10,14}$ En el año 2015, los Centros para el Control y Prevención de Enfermedades (Centers for Disease Control and Prevention, $\mathrm{CDC})^{15}$ han modificado los criterios diagnósticos de las ventriculitis $\mathrm{y}$, actualmente, se requiere, además del criterio microbiológico, manifestaciones clínicas compatibles y la presencia de alteraciones del citoquímico, la tinción de Gram positiva o el aislamiento en hemocultivos, aunque las guías de la Infectious Diseases Society of America (IDSA) de 2017 de ventriculitis y meningitis asociadas a los cuidados de la salud sostienen que el cultivo de LCR es el test más importante para el diagnóstico. ${ }^{4}$

En cuanto a los factores de riesgo, la mayoría de los casos de esta serie ocurrió dentro de los 30 días de colocado el sistema, lo cual coincidió con lo reportado previamente ${ }^{10} \mathrm{y}$ sustentó que el mecanismo involucrado más probable era la colonización del sistema durante el acto quirúrgico. ${ }^{4}$ Cerca de la mitad de los pacientes presentaban el antecedente de uno o más recambios valvulares previos y casi un tercio de infección asociada a shunt previa. Ambos antecedentes han sido asociados al riesgo de una nueva infección en varias series. ${ }^{4,16,17}$ No se encontró una asociación entre el microorganismo actual y el causante de la infección previa; solo 3 pacientes $(5 \%)$ presentaron recurrencia, a diferencia de otros trabajos que reportaron mayor incidencia de recurrencia. ${ }^{3,10}$

La disfunción valvular, sin signos de meningitis, fue la forma de presentación más frecuente, coincidente con la literatura. Frecuentemente, hay solo mínima ventriculitis sin compromiso meníngeo o solo disfunción mecánica producto de la formación del biofilm en el catéter. ${ }^{5}$ En el trabajo de J. K. Lee et al., reportaron un $91 \%$ de pacientes febriles y Turgut et al., un $77 \%,{ }^{11,18}$ mientras que, en nuestra serie, la fiebre estuvo presente en menos de la mitad de los casos. La celulitis del trayecto se presentó solo en 6 infecciones $(11 \%)$, similar a lo reportado por Turgut et al. $(11,4 \%){ }^{18}$

El LCR al ingreso fue patológico en 42 infecciones $(78 \%)$, similar a lo hallado por Conen y col., quienes analizaron 78 infecciones y encontraron que el $80 \%$ presentaba un conteo de leucocitos $>5 \mathrm{cel} . / \mathrm{ml}$ en el LCR, mientras que el $20 \%$ poseía el citoquímico normal. ${ }^{5}$

La presencia de un citoquímico normal, en un paciente con sospecha de infección asociada a SDV-LCR, no la excluye. El cultivo es el gold standard para el diagnóstico de las ventriculitis. En cuanto a la microbiología, se observó un predominio de gérmenes Gram-positivos y una disminución de los gérmenes Gram-negativos, y las infecciones fúngicas fueron raras. Este hallazgo coincide con lo reportado antes por nuestro Centro y recientemente por otros trabajos. ${ }^{9-11,16} \mathrm{En}$ cambio, otros estudios difieren al informar mayor incidencia de bacilos Gram-negativos. ${ }^{19}$

Cerca de la totalidad de las infecciones se 
manejó con tratamiento quirúrgico, que incluyó el retiro del SDV más antibioticoterapia sistémica, coincidente con las recomendaciones y estudios previos que reportaron un mayor éxito con esta estrategia. ${ }^{1,20}$

La mortalidad reportada para las infecciones de los SDV-LCR es de $10 \%$ a $13 \% .{ }^{10,18,21}$ Existen estudios que reportan prevalencias mayores, como el trabajo de, Bathia et al. $(15 \%),{ }^{12}$ Kanik et al. $(15,8 \%),{ }^{10}$ Kim et al. $(17 \%)^{13}$ y Guardado et al. (33\%); ${ }^{22}$ en cambio, otros informan tasas menores, como Ochieng $(4 \%){ }^{14}$ J. K. Lee et al. $(2,85 \%)^{11}$ y Wong et al. $(6 \%) .^{23}$

La mortalidad en nuestra serie fue del 9\%, similar al estudio de Srihawan et al. ${ }^{9}$ Esta variabilidad se ve influenciada, mayormente, por dos factores: el tipo de pacientes que predomina en cada serie (aquellas series con mayor porcentaje de pacientes con tumores del SNC presentan tasas más altas), junto con las series que presentan alta prevalencia de bacilos Gram-negativos resistentes. ${ }^{21,22}$

En el estudio de Gmeiner et al., ${ }^{2}$ quienes analizaron las causas de muerte a largo plazo en pacientes con SDV-LCR, el 78\% de las muertes relacionadas fueron por infección y no por disfunción del corto circuito y sistema, lo que coincidió con el trabajo de Tuli et al., ${ }^{24}$ que evaluaron 907 pacientes pediátricos (excluyeron a los niños con tumores del SNC) y concluyeron que la infección asociada al corto circuito fue un predictor de muerte.

Todas las muertes de esta serie ocurrieron dentro del mes del diagnóstico de la infección; 2 pacientes fallecieron dentro de las 48 horas del ingreso por shock séptico y los 3 restantes fallecieron por progresión de la enfermedad de base asociada a la infección. Luego del análisis univariado, se encontraron como únicos factores asociados a la mortalidad estadísticamente significativos la presencia de hemocultivos positivos, fiebre al ingreso y shock séptico. Escasos estudios realizaron un análisis multivariado de los factores predictores de mortalidad: un estudio de Kim y col., ${ }^{13}$ incluyó a 91 pacientes adultos con una mortalidad de $16,5 \%$ y los factores asociados fueron la falta del retiro del SDV-LCR y el shock séptico. El estudio más importante incluyó a 215 adultos y niños con meningitis y ventriculitis asociadas al cuidado de la salud, ${ }^{9}$ y tuvo como objetivo evaluar los predictores de mala evolución. La mortalidad fue del 9,3\% y, en el análisis de regresión logística, identificó que la edad mayor de 45 años, el examen neurológico anormal y la ventilación mecánica fueron factores de riesgo independientes para un resultado adverso.

Una de las debilidades en nuestra investigación es el tamaño muestral, que puede influir en el poder estadístico para hallar diferencias entre los grupos. Otra limitación del estudio es ser retrospectivo. A su vez, al ser realizado en un centro de alta complejidad, estos resultados podrían no ser extrapolables a otros centros. Su fortaleza es que, de acuerdo con nuestro conocimiento, es uno de los pocos trabajos actualizados de nuestro país en población pediátrica con SDV-LCR que analiza las características de la infección y los factores de riesgo de mortalidad relacionados con este dispositivo.

\section{CONCLUSIONES}

La disfunción valvular, la fiebre y la celulitis del trayecto fueron las manifestaciones clínicas más frecuentes de la infección de SDV-LCR.

El estafilococo coagulasa negativo fue el germen más frecuente. El retiro de la válvula, junto con la antibioticoterapia, fue el tratamiento más utilizado.

La presencia de fiebre al ingreso, los hemocultivos positivos y el shock fueron factores predictores de mortalidad.

\section{REFERENCIAS}

1. Flannery AM, Mitchell L. Pediatric hydrocephalus: systematic literature review and evidence-based guidelines. Part 1: Introduction and methodology. J Neurosurg Pediatr 2014;14(Suppl 1):3-7.

2. Gmeiner $M, W$ agner $H$, Zacher $C$, et al. Long-term mortality rates in pediatric hydrocephalus: a retrospective singlecenter study. Childs Nerv Syst 2017;33(1):101-9.

3. Ram Y, Tina QT. Infections Related to Prosthetic or Artificial Devices. En: Cherry J. Demmler-Harrison G, Kaplan S, et al. Feigin and Cherry's Textbook of Pediatric Infectious Diseases. 7th ed. Philadelphia, PA: Elsevier-Saunders; 2014.Págs.1029-30.

4. Tunkel AR, Hasbun R, Bhimraj A, et al. 2017 Infectious Diseases Society of America's Clinical Practice Guidelines for Healthcare-Associated Ventriculitis and Meningitis. Clin Infect Dis 2017;64(6):e34-65.

5. Conen A, Walti L, Merlo A, et al. Characteristics and Treatment Outcome of Cerebrospinal Fluid ShuntAssociated Infections in Adults: A Retrospective Analysis over an 11-Year Period. Clin Infect Dis 2008;47(1):73-82.

6. Hasbun R. Central Nervous System Device Infections. Curr Infect Dis Rep 2016;18(11):34.

7. Clark R, Lewinski M, Loeffelholz M, et al. Cumitech 31A: Verification and Procedures in the Clinical Microbiology Laboratory. Washington, DC: ASM Press; 2009.

8. Forbes BA, Granato PA. Processing specimens for bacteria. In: Murray P, Baron M, Pfaller F, et al edits. Manual of clinical microbiology. $6^{\text {th }}$ ed. Washington, DC: ASM Press; 1995. Págs.101-3. 
9. Srihawan C, Castelblanco R, Salazar L, et al. Clinical characteristics and predictors of adverse outcomes in adult and pediatric patients with healthcare-associated ventriculitis and meningitis. Open Forum Infect Dis 2016;3(2):ofw077.

10. Kanik A, Sırı N, Kose E, et al. Clinical and Economic Results of Ventriculoperitoneal Shunt Infections in Children. Turk Neurosurg 2015;(1):58-62.

11. Lee JK, Seok JY, Lee JH, et al. Incidence and Risk Factors of Ventriculoperitoneal Shunt Infections in Children: A Study of 333 Consecutive Shunts in 6 Years. J Korean Med Sci 2012;27(12):1563-8.

12. Bhatia PL, Lilani S, Shirpurkar R, et al. Coagulase-negative staphylococci:Emerging pathogen in central nervous system shunt infection. Indian J Med Microbiol 2017;35(1):120-3.

13. Kim H, Kim S, Park G, et al. The causes and treatment outcomes of 91 patients with adult nosocomial meningitis. Korean J Intern Med 2012;27(2):171-9.

14. Ochieng N, Okechi H, Ferson S, et al. Bacteria causing ventriculoperitoneal shunt infections in a Kenyan population. J Neurosurg Pediatr 2015;15(2):150-5.

15. CDC/NHSN Surveillance Definitions for Specific Types of Infections. January 2017. [Acceso: 11 de abril de 2017]. Disponible en: https:/ / www.cdc.gov/nhsn/pdfs / pscmanual/17pscnosinfdef_current.pdf.

16. Rowensztein H, Manfrin L, Paglia M, et al. Características del líquido cefalorraquídeo en niños con infecciones del sistema de derivación ventrículo-peritonea. Arch Argent Pediatr 2015;113(3):244-7.
17. Simon T, Butler J, Whitlock K, et al. Risk factors for first cerebrospinal fluid shunt infection: findings from a multicenter prospective cohort study. J Pediatr 2014;164(6):14628.e2.

18. Turgut M, Alabaz D, Erbey F, et al. Cerebrospinal Fluid Shunt Infections in Children. Pediatr Neurosurg 2005;41(3):131-6.

19. Stenehjem E, Armstrong WS. Central nervous system device infections. Infect Dis Clin North Am 2012;26(1):89-110.

20. Rosanova MT, Paganini H. Infecciones asociadas a sistemas de derivación de líquido cefalorraquídeo. Med Infant 1997;4(1):26-30.

21. Sacar S, Turgut H, Toprak S, et al. A retrospective study of central nervous system shunt infections diagnosed in a university hospital during a 4-year period. BMC Infect Dis 2006;6:43.

22. Rodríguez Guardado A, Blanco A, Asensi V, etal.Multidrugresistant Acinetobacter meningitis in neurosurgical patients with intraventricular catheters: assessment of different treatments. J Antimicrob Chemother 2008;61(4):908-13.

23. Wong HT, Pu JK, Wong WK, et al. Primary ventriculoperitoneal shunting outcomes: a multicentre clinical audit for shunt infection and its risk factors. Hong Kong Med J 2016;22(5):410-9.

24. Tuli S, Tuli J, Drake J, et al. Predictors of death in pediatric patients requiring cerebrospinal fluid shunts. J Neurosurg 2004;100(5 Suppl Pediatrics):442-6.

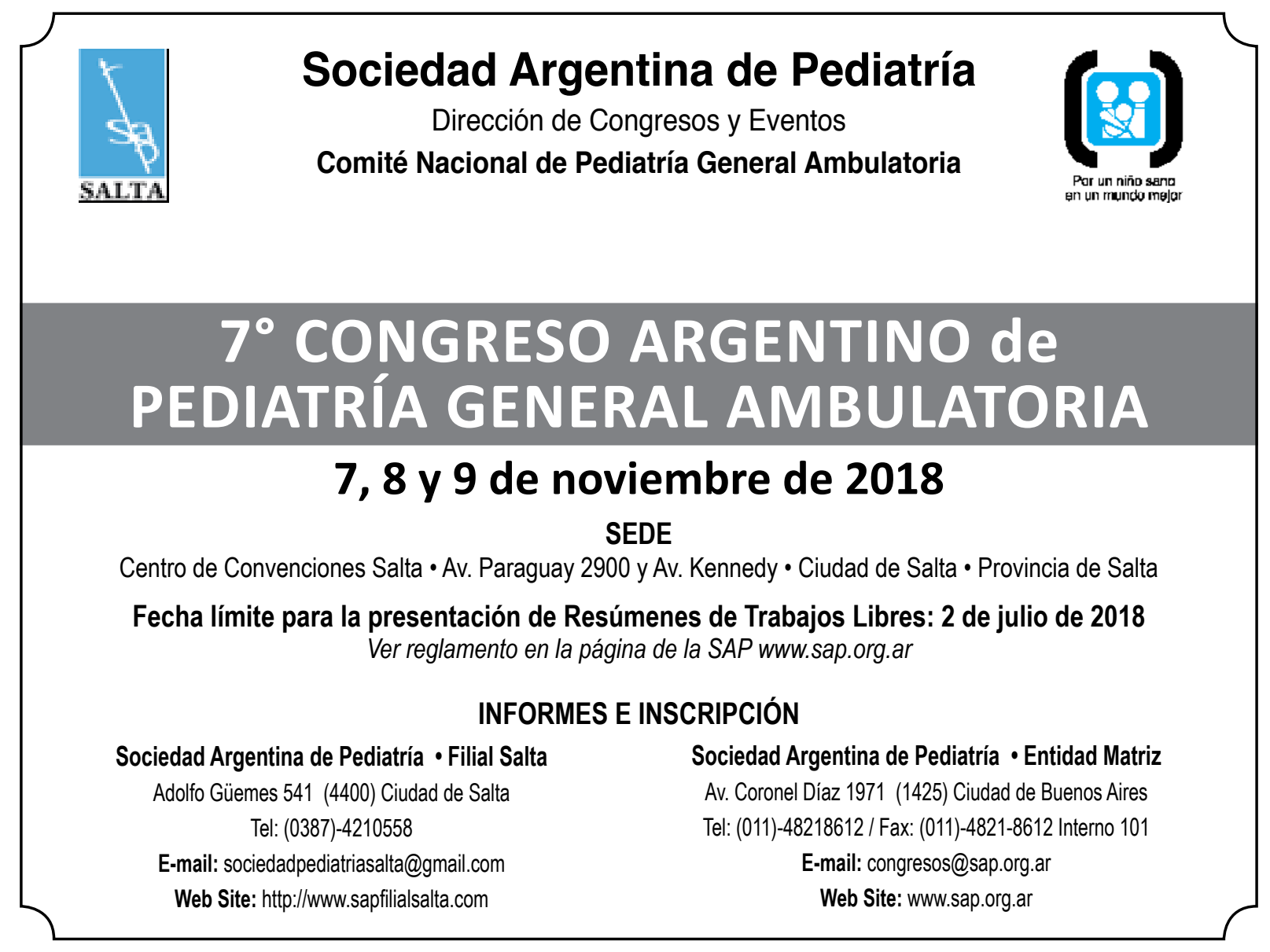

